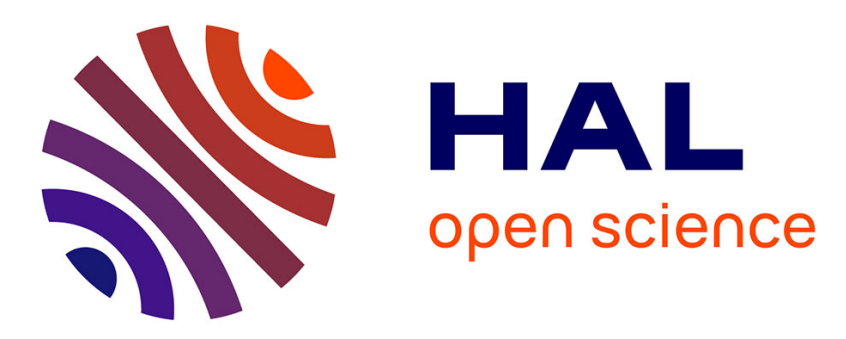

\title{
Assistance for Older Adults in Serious Game using an Interactive System
}

Minh Khue Phan Tran, François Bremond, Philippe Robert

\section{To cite this version:}

Minh Khue Phan Tran, François Bremond, Philippe Robert. Assistance for Older Adults in Serious Game using an Interactive System. GALA 2015 - 4th International Conference Game and Learning Alliance Conference, the Serious Games Society and the University of Genoa, Dec 2015, Rome, Italy. pp.6, 10.1007/978-3-319-40216-1_30. hal-01275258

\section{HAL Id: hal-01275258 https://hal.science/hal-01275258}

Submitted on 19 Feb 2016

HAL is a multi-disciplinary open access archive for the deposit and dissemination of scientific research documents, whether they are published or not. The documents may come from teaching and research institutions in France or abroad, or from public or private research centers.
L'archive ouverte pluridisciplinaire HAL, est destinée au dépôt et à la diffusion de documents scientifiques de niveau recherche, publiés ou non, émanant des établissements d'enseignement et de recherche français ou étrangers, des laboratoires publics ou privés. 


\title{
Assistance for Older Adults in Serious Game using an Interactive System
}

\author{
Minh Khue PHAN TRAN ${ }^{1,2,3}$, François BREMOND ${ }^{1}$, Philippe ROBERT ${ }^{2}$ \\ ${ }^{1}$ Stars, Inria, Sophia Antipolis, France. \\ Francois.Bremond@inria.fr \\ ${ }^{2}$ CoBTek Cognition Behaviour Technology EA 7276, \\ Research Center Edmond and Lily Safra, \\ University of Nice Sophia Antipolis, Nice, France. \\ probert@unice.fr \\ ${ }^{3}$ Genious Interactive, 3 ter rue des Pins,Montpellier, France. \\ m.phantran@genious.com
}

\begin{abstract}
Serious Games offer a new way to older adults to improve various abilities such as the vision, the balance or the memory. However, cognitive impairment causes a lot of difficulties to them when actively practicing these games. Their engagement and motivation are reduced rapidly when encountering successive problems without any help. In this paper, we present an approach to assist older adults in Serious Game using an interactive system. Three groups of players with different cognitive impairments (Mnesic Plaint, MCI and Alzheimer) have been tested with the system in a concentration-based game. As the experimental results, the players performed a high performance when playing with the assistance of the system, especially among Alzheimer group. The future work aims to perform this approach with a larger population and explore other factors which can influence on the players' motivation.
\end{abstract}

\section{Introduction}

In recent years, many researches proved that the video game, renamed under a special term «serious game», can boost mental skills for older adults [1], [2]. Moreover, many projects offer games in some specific problematics of them [3], [4].

This new technology can maintain motivation and improve engagement's player. However, there is a paradox among the older players: they could quickly discourage because of many difficulties due to lack of game culture or their fragile memory. Consequently, they cannot play alone and could abandon the game after a short use. The big challenge here is actively keeping them in game as long as possible by maintaining permanently their motivation and their engagement. For that, detecting their motivation level is necessary for determining the moment to help them.

The paper is organized as follows. In section 2, we make an overview of many approaches related to player's engagement and motivation. Section 3 describes in details our approach. In Section 4, we present an experimental application of the system with 30 persons presenting the cognitive impairment (Mnesic, MCI and 
Alzheimer) through a concentration-based game. In the last section, we discuss a conclusion and future works to be presented later.

\section{Related Work}

In the case of the use of gamification into the non-gaming context [5], intrinsic motivation and extrinsic motivation [6] exist together. But when the player is an older adult, intrinsic motivation is more important than extrinsic motivation.

Some works have been conducted to adjust the game design [7, 8] and the gameplay [9] as well as introduce new devices [10,11] in the games. Also, a new source of motivation through the robot is slowly exploited. In Japan, animal therapy has been replaced by robot therapy in assisted activities [12] as well as improvement and prevention dementia for older adults. In the case of cognitive training, a socially assistive robot in [13] has been tested with patients with dementia in a specific music cognitive-base game. Some systems in Human-Machine Interaction estimate the attention's level of the user based on various factors such as face's orientation [14] or several backchannels' behaviours [15].

However, these approaches do not handle the difficulties generated by cognitive impairment such as lack of memory or distraction during the game. They cannot recognize the moment that the older players are distracted when they realize many wrong handlings or forgets the rules and do not know how to continue to play.

\section{Interactive System}

We have proposed an interactive system in order to provide several assistances to older adults. In [16], the proposed system interacts with the user in terms of his movements and his position in the related zones. In this paper, the system will focus on the gestures performed by older adults when they are playing the game in order to determine the situation for giving him help.

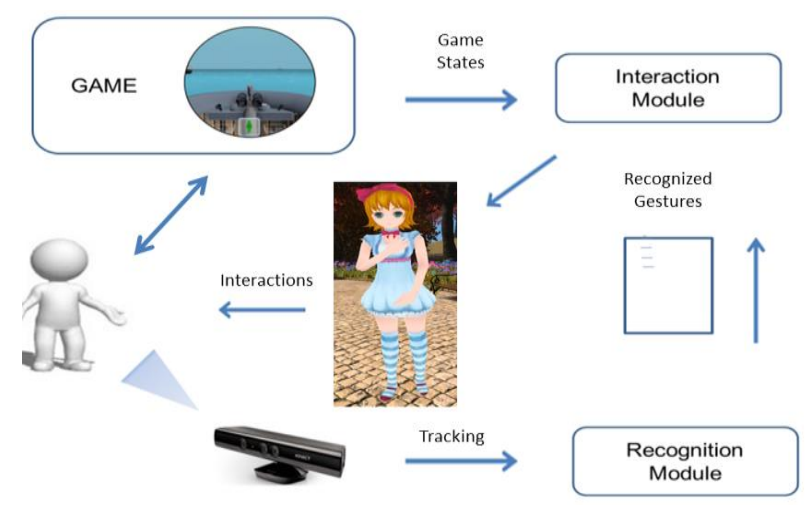

Fig. 1. Structure of the interactive system 
Figure 1 describes the structure of the interactive system, which is a combination of two modules: Recognition Module and Interaction Module. Thanks to the Kinect camera of Microsoft and the supplied Kinect SDK, the Recognition Module tracks and recognizes the player's gestures then sends them to the Interaction Module. The last one collects these information and combines with game states provided by the game in order to determine the type of interaction with the player through a $3 \mathrm{D}$ animated avatar. Here, the avatar can move, realize many expressions and give the verbal communications to player. The interest of a virtual avatar is demonstrated in the literature. Indeed, he can bring to players a feeling of companion [17], increase his attention and his sympathy [18] and more importantly, improve his interaction with the system [19].

\section{Experiment}

The aim of this experiment is to evaluate the efficiency of the proposed system for keeping actively the players in the game. Three groups of ten older adults (Tab. 1), categorized by their cognitive state (Mnesic plaint, MCI, Alzheimer), are solicited, by the doctor, to perform a cognitive training session through a game during their memory consultation.

Our hypothesis is that the proposed system can improve their performance as well as possible during the game.

\subsection{Protocol}

The session takes place in a private room, equipped with a screen, a game with Camera Kinect (Fig .2) and the presence of a therapist. Each session lasts approximately 20 minutes. In short, there are three phases of the game:

- Phase 1: the player learns rapidly how to play the game with the therapist.

- Phase 2: the player plays the game alone without any helps.

- Phase 3: the player plays the game with the avatar's assistances.

The order of phases for one session is as follows: all the players begin with phase 1. Then, a randomization is made to determine between phase 2 and 3 in which one if the players continue as described in Figure 3.

Players' performance is collected only in phase 2 and 3. It's presented by:

- Playing time of a phase: the duration when the players start the phase until the end of the phase.

- Score for the current phase. 


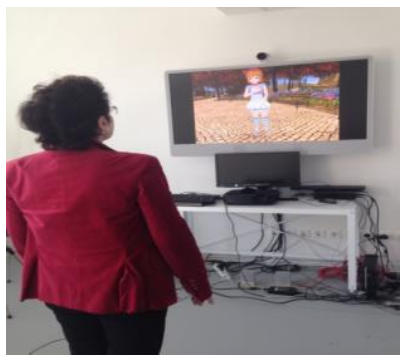

Fig. 2. Player interacts with the avatar

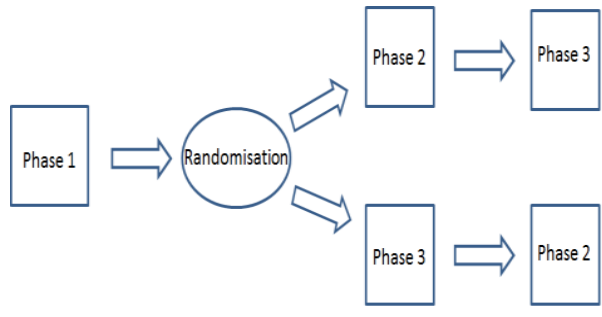

Fig. 3. Order of the phases for each player

\subsection{Adaptation of System}

In this experimentation, we designed the game in the simple manner so that the players can browse the menu, choose the level, follow the tutorial and play the game by only raising hands (left /right). Moreover, we elaborated all of communication texts with a psychologist in order to create a positive ambiance and encourage the players. In addition, we provide 3 types of assistance to the players:

- Assistance against inactivity: when the players do not interact with the game or the avatar for a while, the avatar recalls the players' attention and gives some expected guidelines according to the current game state.

- Assistance against errors: during the game, if the players make a lot of errors, the avatar supposes that they can forget the rules then recall them.

- Assistance for tutorial: when the players desire to realize a tutorial before playing the game, the avatar guides them basing on their gestures.

\subsection{Experimental Results}

Obviously, the players always finish the game and have a play time which is shorter in phase 3 than in phase 2 (Fig. 4,5 ). More importantly, two players in Alzheimer group abandon the game in phase 2. Indeed, without any help, the players spend much time to recall the rules of game, while in phase 3, thanks to the assistance against inactivity the players can continue and finish the game. The case of two abandoned players emphasizes the interest of assistance against inactivity. Their motivation tumbles down quickly when they cannot remember the rules of game.

The score of the players benefits also the assistance of system (Fig. 4, 5). Thanks to the assistance against errors, the players can reduce the number of errors. Especially, Alzheimer group had a score more important than other groups.

In order to understand how the players appreciate the assistance of the system, we asked a short questionnaire at the end of each training session, concerning various characteristics of the avatar (voice, communication's text, appearance, animation and intervention). All of the players agree that avatar's voice is clear and the 
communication is understandable. 27 players think that the appearance is pleasant and the other three find it normal. Moreover, avatar's animation is judged natural by 26 players, normal by 2 players and still factice by the last two. Globally, the players appreciate positively the avatar as well as its interactions during the game.
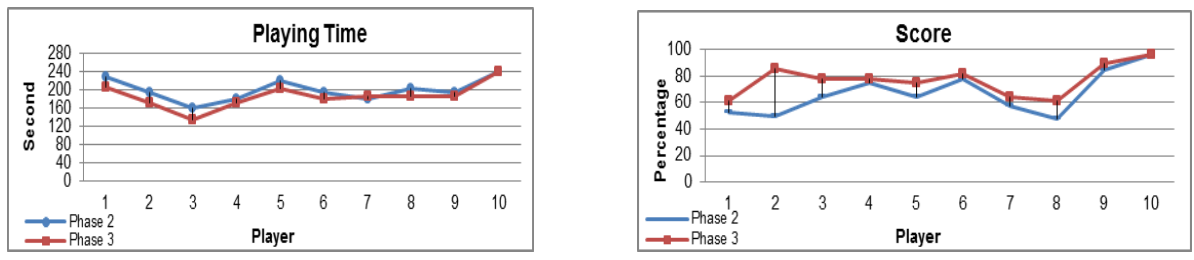

Fig 4. The results of MCI group in phase 2 and phase 3
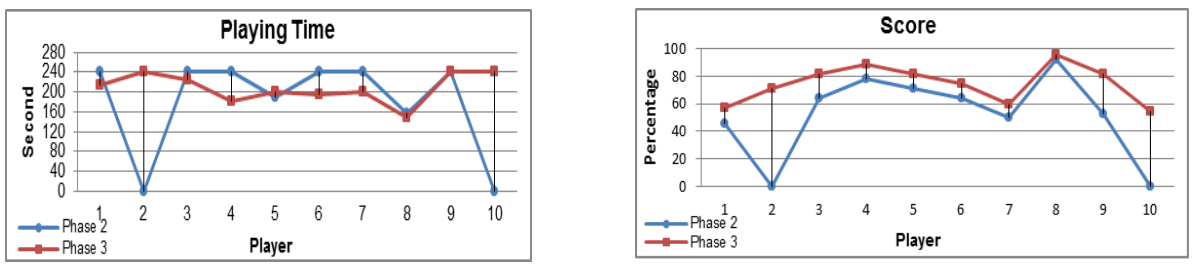

Figure 5. The results of Alzheimer group in phase 2 and phase 3

\section{Discussion and Conclusion}

In this paper, we proposed an assistive system for older adults with the Serious Game. Because of the decline of their cognitive functionalities, they have a lot of difficulties to perform a successful session. They need real-time assistance. The more they are helped, the longer their motivation is maintained. An experiment was conducted with 30 older players categorized by their diagnostic (Mnesic Plaint, MCI and Alzheimer) during the memory consultation. The proposed system aims to maintain their motivation by keeping them actively and improving their performance during a cognitive training game. Therefore, the system uses a 3D animated avatar to give assistances (recalls, guidelines) according to the inactivity and the number of errors. As the experimental results, the players realized a high performance and use effectively the rules and guidelines of the game thanks to the assistances of the avatar. Future work consists in confirming these results with a larger population on one hand, and on the other hand, analysing more precisely the reason of errors made by the players. In addition, it could be also interesting to explore more factors which can influence their motivation (e.g. gameplay, music, new kind of assistance). 


\section{References}

1. Belchior, P. D. C.: Cognitive training with video games to improve driving skills and driving safety among older adults. Dissertation Abstracts International: Section B: The Sciences and Engineering, 68(9-B) (2008)

2. Anguera, J. A., Boccanfuso, J., Rintoul, J. L., Al-Hashimi, O., Faraji, F., Janowich, J., \& Gazzaley, A.: Video game training enhances cognitive control in older adults. Nature, 501(7465) (2013) 97-101

3. VERVE project (http://verveconsortium.eu/)

4. Az@Game project (http://www.azagame.fr/)

5. Sebastian, D., Miguel, S., Lennart, N., Kenton, O'., Dan, D.: Gamification : using gamedesign elements in non-gaming contexts, CHI '11 Extended Abstracts on Human Factors in Computing Systems, Canada (2011)

6. Edward, L. Deci., Richard, M. R.: Intrinsic Motivation and Self-Determination in Human Behavior. Springer (1985)

7. Bruno, B., Frédérick, I., Abdenour, B., Bob-Antoine J. M.: Developing serious games specifically adapted to people suffering from alzheimer, Proceedings of the Third international conference on Serious Games Development and Applications, Germany (2012) 243-254

8. Ji-won, L., Seong-jun, P.: A Study on Interface Design of Serious Game for the Elderly, in Advanced Science and Technology Letters Vol.39 (2013) 159-163

9. Tong, T., Chignell, M., Lam, P., Tierney, M. C., \& Lee, J.: Designing Serious Games for Cognitive Assessment of the Elderly. In Proceedings of the International Symposium of Human Factors and Ergonomics in Healthcare, Vol. 3, SAGE Publications (2014) 28-35

10. Kretschmann, R., Dittus, I., Lutz, I., and Meier, C.: Nintendo Wii Sports: Simple gadget or serious measure for health promotion? - A pilot study according to the energy expenditure, movement extent, and student perceptions. Proceedings of the GameDays 2010 - Serious Games for Sports and Health, Darmstadt (2010) 147-159

11. D. Webster and O. Celik.: Systematic review of Kinect applications in elderly care and stroke rehabilitation,J. Neuroeng. Rehabil., vol. 11, no. 1 (2014) 108

12. K. Wada, T. Shibata, T. Saito, and K. Tanie.: Effects of Robot Assisted Activity for Elderly People and Nurses at a Day Service Center, Proc. of the IEEE, Vol.92, No.11 (2004) 17801788

13. Tapus, A., Ţăpuş, C., \& Matarić, M. J.: The use of socially assistive robots in the design of intelligent cognitive therapies for people with dementia. In Rehabilitation Robotics IEEE International Conference (2009) 924-929

14. R. Ishii, Y. I. Nakano, and T. Nishida : Gaze awareness in conversational agents: Estimating a user's conversational engagement from eye gaze. In ACM Transactions on Interactive Intelligent Systems (2013) 11:111:25

15. A.Vinciarelli, M. Pantic, D. Heylen, C. Pelachaud, I. Poggi, F. D'Errico, and M. Schroeder: Bridging the gap between social animal and unsocial machine: A survey of social signal processing. In IEEE Transactions on Affective Computing (2012) 69-87

16. Minh Khue, P.T., François, B., Philippe, R.: Comment intéresser les personnes âgées aux Serious Game ?. JA-SFTAG 2014, France (2014)

17. Martin, M. M., Andreas, H., Sascha, F., and Siegfried, W.: Avatars in assistive homes for the elderly. HCI and usability for education and work. Springer (2008) 391-402

18. Laura, P. V., Lazlo R., Barbara B., Candace L. S., Timothy B.: Designing relational agents as long term social companions for older adults. In he 12th international conference on Intelligent Virtual Agents (2012)

19. Amalia, O., María, D. P. C., David, O., Jose, J. Y., Cristina, B., M. Feli Gonzalez, Igone, E.: Elderly users in ambient intelligence: does an avatar improve the interaction?. In the 9th conference on User interfaces for all (2006) 